\title{
A Medida do Talento: Evidências de Validade de uma Escala para Aferir Talento em Organizações
}

\author{
Manoela Ziebell de Oliveira ${ }^{1}$ \\ Jean Carlos Natividade \\ Programa de Pós-Graduação em Psicologia da Universidade Federal do Rio Grande do Sul, \\ Porto Alegre, Brasil \\ William Barbosa Gomes \\ Instituto de Psicologia da Universidade Federal do Rio Grande do Sul, Porto Alegre, Brasil
}

\section{Resumo}

A alta competitividade no mundo dos negócios tem levado organizações a uma disputa agressiva por profissionais de alto talento. Essa disputa tem gerado debates, tanto no âmbito organizacional quanto no âmbito acadêmico, sobre como definir o fenômeno e acessar sua ocorrência na população. Neste estudo revisou-se a literatura referente à conceituação de talento, e formulou-se uma definição mensurável do construto, extensiva a diversos contextos organizacionais. Elaborou-se um conjunto de itens referentes a três dimensões do talento: qualidade, velocidade e autonomia na realização do trabalho. Em seguida, buscaram-se evidências de validade e precisão da medida elaborada. Participaram do teste empírico do instrumento 308 profissionais ativos no mercado de trabalho, 61\% homens, em média 35,1 anos de idade. Os participantes responderam um questionário contendo 20 itens referentes ao talento, uma escala de personalidade no modelo dos cinco grandes fatores e questões sobre experiência profissional. Análises exploratórias e confirmatórias, em acordo com a definição do construto, corroboraram a estrutura de três fatores do instrumento. Os índices de precisão de todas as dimensões foram satisfatórios, coeficientes alfa superiores a 0,80 . As correlações observadas entre os fatores do talento, de personalidade e experiência profissional fornecem adequadas evidências de validade para o instrumento. Pessoas com altos níveis de talento apresentam escores altos em conscienciosidade, foram promovidas mais frequentemente, e receberam ofertas de trabalho de outras empresas. Discutem-se as possibilidades de uso do instrumento na área acadêmica e em situações aplicadas.

Palavras-chave: Talento, desempenho no trabalho, avaliação de recursos humanos, desempenho.

\section{The Measure of Talent: Evidence of the Validity of a Scale to Measure Talent in Organizations}

\begin{abstract}
A great competitiveness in business has led organizations to an aggressive race for top talent professionals. This dispute has generated debates, in both the organizational, and the academic field, about how to define the phenomenon and how to assess its occurrence in the general population. This study reviewed the literature about talent, and proposed a measurable definition of the construct, which can
\end{abstract}

Endereço para correspondência: Instituto de Psicologia, Laboratório de Fenomenologia Experimental e Cognição, Rua Ramiro Barcelos, 2600, sala 123, Bairro Santa Cecília, Porto Alegre, RS, Brasil 90035-003. E-mail: manoela.ziebell@gmail.com, jeannatividade@gmail.com e wbgomes@gmail.com Os autores receberam apoio financeiro da Comissão de Aperfeiçoamento de Pessoa de nível Superior (CAPES) durante o período de realização desta pesquisa. 
be extended to various organizational contexts. We developed a set of items related to three dimensions of talent: quality, speed and autonomy when performing the work. We then searched for evidence of validity and reliability of the measure. Participants on the empirical test of the instrument were 308 professionals active in the labor market, $61 \%$ men, mean age 35.1 years. The participants answered a questionnaire containing 20 items related to talent, a personality scale based on the Big Five factors and questions about employment history. Exploratory and confirmatory analyzes, in accordance with the definition of the construct, supported the three-factor structure of the instrument. Reliability indexes of all dimensions were satisfactory, showing alpha coefficients above .80 . The observed correlations between talent and personality factors and professional experience provide adequate evidence of validity of the instrument. Individuals with high levels of talent scored high in conscientiousness, received promotions more often and had job offers from other companies. We discuss the possibilities of using the instrument in academic and applied situations.

Keywords: Talent, job performance, personnel evaluation, performance.

\section{La Medida del Talento: Evidencias de la Validez de una Escala para Medir el Talento en las Organizaciones}

\section{Resumen}

La alta competitividad en los negocios ha llevado las organizaciones a una disputa agresiva por los profesionales de gran talento. Esta disputa ha generado debates, tanto en el contexto organizacional como en el contexto académico, acerca de cómo definir el fenómeno y estudiar su incidencia en la población. Los autores examinaron la literatura sobre el concepto de talento, y concibieron una definición mensurable del constructo, que se puede extender a diversos contextos organizacionales. Generaron una serie de ítems relacionados con tres dimensiones de talento: calidad, rapidez e independencia en la realización del trabajo. Posteriormente buscaron pruebas de validez y confiabilidad de la medida elaborada. Participantes del test empírico del instrumento fueron 308 profesionales activos en el mercado laboral, $61 \%$ hombres, edad media 35,1 años. Los participantes respondieron un cuestionario con 20 ítems relacionados con talento, una escala de la personalidad en el modelo de los cinco grandes factores y preguntas acerca de la experiencia profesional. Los estudios exploratorio y confirmatorio, de acuerdo con la definición del constructo, fueron compatibles con la estructura de tres factores del instrumento. Los índices de confiabilidad de todas las dimensiones fueron satisfactorios, coeficientes alfa superiores a 0,80 . Las correlaciones observadas entre los factores de talento, personalidad y experiencia profesional proporcionaron pruebas suficientes de la validez del instrumento. Las personas con altos niveles de talento tienen puntuaciones altas en responsabilidad, reciben promociones con mayor frecuencia y recibe ofertas de empleo de otras empresas. Se discuten las posibilidades de utilización del instrumento en situaciones académicas y aplicadas.

Palabras clave: Talento, rendimiento laboral, evaluación de recursos humanos, desempeño.

Mudanças no mundo dos negócios, como o aumento de capital financeiro e o fácil acesso a novas tecnologias, têm fomentado nas organizações a busca por novos diferenciais para alcançar resultados competitivos. Uma estratégia adotada por muitas delas tem sido ampliar os investimentos no capital humano (Martin \& Schmidt, 2010). Como consequência, as táticas para atrair e reter os melhores trabalhadores vem tornando-se tão agressivas que profissionais do mercado e pesquisadores têm se referido a elas como uma "guerra por talentos" (Aguinis, Gottfredson, \& Joo, 2012).

O termo "guerra por talentos" foi introduzido em 1998, quando a maior consultoria de gestão de negócios dos Estados Unidos à épo- 
ca, McKinsey e Company, publicou os resultados de uma pesquisa institucional que concluía que para reter os mais altos talentos era preciso "lutar" por eles. Isso envolveria, segundo os autores, adotar medidas ousadas para manter os profissionais na organização: alterar políticas salariais, permitir maior participação na tomada de decisões estratégicas, viabilizar e facilitar seu desenvolvimento profissional, entre outras (Chambers, Foulon, Handfield-Jones, Hankin, \& Michaels, 1998).

Em sua publicação, Chambers et al. (1998) não definiram o que era o alto talento, mas sugeriram que cada companhia, individualmente, definisse os padrões-ouro. Adicionalmente, apresentaram algumas características que poderiam ajudar a identificar os profissionais como tal. Altos talentos seriam caracterizados como indivíduos que agregam competitividade às organizações, por se tratar de recursos humanos privilegiados. Eles são pagos tanto pelo trabalho que realizam como pela singularidade que oferecem. No entanto, os altos talentos apresentam alta mobilidade e, com frequência, assumem contratos psicológicos de curto prazo com as organizações. Apesar das indicações dos autores, uma lacuna em definir talento de forma objetiva e mensurável vem dificultando a identificação destes indivíduos pelas organizações. (Pepermans, Vloeberghs, \& Perkisas, 2003). Como consequência, muitas delas passaram a utilizar o "talento" como sinônimo de força de trabalho (Dries \& Pepermans, 2008; Ready, Conger, \& Hill, 2010).

De fato, o talento não é um fenômeno que apresente definições consensuais (Michaels, Handfield-Jones, \& Axelrod, 2001; Pepermans et al., 2003). Em função da diversidade de perspectivas sobre o assunto, os estudos produzidos apresentam ampla variação de embasamentos teóricos e escassez de instrumentos adequados para acessar o construto. Atentos para essas fragilidades, os autores do presente estudo têm dois objetivos principais: (a) apresentar as principais perspectivas sobre o conceito de talento, suas implicações, e os desafios que apresentam às organizações que as adotam e (b) apresentar uma medida de talento capaz de acessar esse construto em diferentes contextos e organizações.
Após uma revisão exaustiva e não sistemática dos termos alto talento, alto potencial e alto desempenho, em periódicos nacionais, internacionais, PsychINFO e no Google Scholar, entre os anos de 1960 e 2013, organizou-se a produção científica encontrada em quatro grupos. São eles: talento como fenômeno inato; talento como resultado de prática deliberada; talento como produto de forças individuais; e talento como produto de habilidades individuais.

\section{Talento como Fenômeno Natural (ou Dotação)}

Essa forma de conceber talento teve origem com os trabalhos de Galton (1869), que propunham a soberania dos aspectos hereditários em relação aos aspectos ambientais, na determinação da inteligência superior. Por muito tempo, pesquisadores identificados com essa proposição definiram operacionalmente o talento a partir de escores superiores em avaliações de aptidões e de inteligência (Nakano \& Siqueira, 2012). Dada tamanha influência, ainda hoje muitos pesquisadores e profissionais entendem o talento como sinônimo de inteligência e superdotação (Nakano \& Siqueira, 2012).

Em meados da década de 1980, Gagné (1985) propôs uma distinção entre os termos dotação e talento. Segundo essa proposição, dotação corresponderia à competência que é distintamente superior à média em um ou mais domínios de habilidade e aptidão; talento, por outro lado, corresponderia ao desempenho distintamente superior à média em um ou mais campos do desempenho humano. Segundo essa proposição, todo o indivíduo talentoso é dotado de habilidades excepcionais, enquanto o contrário não é verdadeiro (Gagné, 1985).

Atualmente, os pesquisadores sob essa perspectiva compreendem o talento em termos de desempenho superior e habilidade notável expressos em comportamentos, ações e atitudes visíveis e passíveis de análise (Gagné, 2009). Assim, a emergência do talento só é possível quando há capacidade natural ou potencial para a ação, e condições ambientais favoráveis à área de atividade (Gagné, 2009). Nessas condições, o desempenho aumentaria uniformemente como 
uma função da prática até alcançar um ponto máximo em que estagnaria (Ericsson \& Charnes, 1994). Como decorrência, treinamentos poderiam afetar apenas alguns dos componentes do desempenho, mas os componentes inatos não poderiam ser modificados. Assim, em última análise, fatores determinados geneticamente seriam as principais variáveis explicativas de um alto talento (Ericsson \& Charnes, 1994).

No campo acadêmico a maior concentração de estudos que adotam essa abordagem é encontrada nas áreas da educação, do estudo da inteligência, e da dotação. As principais estratégias empregadas para acessar os aspectos inatos do talento são os testes de habilidade e aptidão, e experimentos envolvendo medidas de tempo de reação para a detecção de estímulos sensoriais e testes de memória. O uso dessas estratégias, contudo tem se mostrado pouco eficiente em discriminar níveis de desempenho em tarefas complexas e ainda menos eficiente para predizer o desempenho em domínios específicos em situações de vida real (Ericsson \& Charnes, 1994).

Boa parte dos testes de habilidade e aptidão desenvolvidos a partir da concepção de talento como fenômeno inato, destinam-se a avaliação de crianças em idade escolar. Alguns exemplos são: a Gifted Rating Scale - School Form (Nakano \& Siqueira, 2012), e o Munich High-Ability Battery Test (Heller \& Perleth, 2008). O primeiro consiste na avaliação de habilidades como raciocínio lógico e abstrato de estudantes entre seis a 14 anos, pelos professores. O segundo avalia preditores cognitivos (por exemplo, habilidades intelectuais, criativas e sociais), não cognitivos (por exemplo, personalidade), e sociais (por exemplo, interesses motivações, autoconceitos) dos altos talentos.

Em termos práticos, a tendência em organizações que concebem o padrão-ouro do talento como fenômeno natural é buscar profissional cujas características atendam de pronto as suas necessidades competitivas. Elas procuram as "estrelas solitárias," isto é, aqueles profissionais que pela combinação entre alto talento e habilidades superiores são bem sucedidos em outras organizações (Groysberg, Lee, \& Abrahams, 2010). As empresas oferecem a essas estrelas salários superiores à média para que ocupem posições estratégicas e depositam nelas as expectativas de sucesso organizacional (Martin \& Schmidt, 2010). Para as organizações, o risco em adotar tal perspectiva é o de subestimarem o efeito da cultura organizacional nos resultados do profissional ao priorizar características cognitivas dos indivíduos. Além disso, quando as organizações não são capazes de oferecer ao alto talento o nível de suporte de que ele dispunha em uma organização anterior, há grandes chances de que, mesmo recebendo altos salários, seu desempenho seja insatisfatório (Groysberg, McLean, \& Nohria, 2006).

\section{Talento como Resultado da Prática Deliberada (ou Expertise)}

Essa forma de conceber talento foi influenciada pelos trabalhos de DeGroot (1965) que investigavam o impacto do treinamento sobre os processos cognitivos de enxadristas de alto talento. Estudos subsequentes indicaram que a principal variável explicativa da diferença entre o desempenho de novatos e especialistas era a prática intensa do último grupo em domínios específicos de atuação (Simon \& Chase, 1973). Posteriormente, essa prática intensa cujo resultado é o rendimento superior, passou a ser chamada de prática deliberada (Ericsson \& Lehman, 1996).

A prática deliberada, segundo seus proponentes diz respeito ao esforço envolvido em melhorar os níveis atuais de desempenho. Tem como principais características: concentração absoluta, descoberta de estratégias de solução de problemas mais efetivas, aprimoramento das formas de desempenhar as tarefas (Ericsson \& Lehman, 1996), e o desenvolvimento ao longo de três etapas. Na primeira o indivíduo é considerado iniciante e precisa seguir instruções dos orientadores e aplicar regras e procedimentos passo-a-passo. Como resultado da prática e da aquisição de conhecimento sobre a atividade, o aprendiz torna-se mais capaz de gerar os mesmos resultados de forma mais rápida e eficiente. Por fim, torna-se especialista, passando a ser capaz de responder de forma rápida e intuitiva (Ericsson, 2008). 
Aqueles que se fundamentam nessa perspectiva defendem que para um indivíduo concluir a última etapa e se tornar especialista, são necessários, no mínimo, 10 anos de exercício recorrente ou 10.000 horas de prática (Ericsson, 2004; Ericsson, Roring, \& Nandagopal, 2007). Os resultados do treinamento podem ser facilitados (mas não acelerados) quando o indivíduo apresenta disponibilidade, motivação, tem acesso a mentores especializados e materiais adequados e inicia cedo na área de atuação (Ericsson, 2004).

No campo acadêmico, a maior concentração de estudos que adotam essa abordagem é encontrada na área da música e dos esportes. As principais estratégias empregadas pelos pesquisadores para acessar os aspectos aprendidos do talento envolvem avaliação de programas de treinamento de habilidades em experimentos controlados (Ericsson, 2008; Ericsson \& Williams, 2007). Outros estudos sob essa perspectiva têm sido realizados com escalas de autorresposta sobre a motivação para a prática deliberada. Alguns exemplos são: o Work and Family Orientation Questionnaire (WOFO) que avalia o quanto um indivíduo está disposto a trabalhar duro visando a ter bom desempenho (dimensão Trabalho); o quanto o indivíduo é orientado por desafios para ter um bom desempenho em tarefas difíceis (dimensão Maestria); e o quanto se interessa por competir e se dedicar a vencer (dimensão Competição; Helmreich \& Spence, 1978); o Deliberate Practice Motivation Questionnaire (DMQ) foi desenvolvido a partir do WOFO com a finalidade de avaliar a motivação de enxadristas para realizar a prática deliberada do esporte (DeBruin, Rikers, \& Schmidt, 2007).

Organizações que apresentam a cultura orientada por essa perspectiva e entendem o talento como resultado da prática deliberada tendem a buscar profissionais graduados que tenham exercitado seus conhecimentos anteriormente em cargos semelhantes (Groysberg et al., 2010). Esses profissionais são então treinados como aprendizes por profissionais experientes, até que possam ganhar as credenciais para a prática de forma independente (Ericsson, 2008).
Um exemplo desses treinamentos são os programas de trainee, de mentoring e de coaching, em que os profissionais são submetidos a situações críticas e reais, recebendo orientações e feedbacks de colegas mais experientes visando a melhores resultados.

A eficácia da prática deliberada está associada a atividades de curta duração, com oportunidades para feedbacks imediatos, reflexão e correções (Ericsson, 2004). Por isso, organizações que desenvolvem treinamentos para talentos sem levar em conta tais fatores associados à aprendizagem correm o risco de submeter os profissionais a programas excessivamente exaustivos ou desnecessários. A razão para tal é que o envolvimento na prática deliberada demanda muito do profissional, e não é, por si só, uma atividade prazerosa e instigante (Ericsson, 2004). Por isso características individuais, como interesse pelo trabalho e desejo pelo sucesso, são fatores que influenciam a forma como os profissionais se dedicarão aos treinamentos (Garcia-Santos, Almeida, \& Werlang, 2012).

\section{Talento como Produto de Forças}

Essa forma de conceber talento iniciou-se com o trabalho de Bloom (1985), segundo o qual qualquer indivíduo tem um grande potencial em pelo menos um domínio específico. O desafio para os psicólogos, segundo ele, é descobrir a forma de desvendar esse potencial e possibilitar que os indivíduos se destaquem em atividades pelas quais mais se interessam e das quais mais desfrutam (Bloom, 1985). Esses resultados levaram outros pesquisadores a testar a hipótese de que os indivíduos têm maior potencial para crescer em áreas nas quais apresentam um bom desempenho do que naquelas em que apresentam fraquezas.

Atualmente, sob esse ponto de vista, o talento é entendido como um padrão natural de pensamentos, sentimentos e comportamentos que pode ser acessado e utilizado de forma produtiva (Hodges \& Clifton, 2004). Esse padrão é desenvolvido principalmente entre os três e os 15 anos de idade, época em que as principais estruturas cerebrais se formam e são reforçadas devido à 
prática em alguns domínios (Coffman \& Gonzalez-Molina, 2002). Deste modo, a prática seria capaz de especificar quais seriam os domínios ou forças pessoais de um indivíduo no futuro. Apesar do estabelecimento de padrões ocorrer precocemente, a possibilidade de novos circuitos neurais serem desenvolvidos ao longo da vida não é descartada. Nessa perspectiva o investimento mais promissor para transformar forças pessoais em talento envolveria investir no exercício dos domínios mais explorados ao longo da vida (Hodges \& Clifton, 2004).

No campo acadêmico aspectos explorados pela Psicologia Positiva apresentam afinidades com essas proposições (Seligman \& Csikszentmihalyi, 2000). Duas estratégias empregadas pelos pesquisadores para acessar o talento são o The Values in Action (VIA) Classification of Strengths (Peterson \& Seligman, 2004), e o StrengthsFinder (Hodges \& Clifton, 2004). A primeira estratégia é um questionário de autorresposta no qual os indivíduos são solicitados a indicar em que grau as afirmativas referentes a diferentes forças se aplica a eles. A segunda é uma avaliação da previsibilidade dos padrões de comportamento dos indivíduos. O relatório resultante da aplicação desse último instrumento apresenta um mapa das principais forças e sugestões de como desenvolvê-las no indivíduo.

Em termos práticos, organizações onde o padrão de alto talento é visto como produto das forças individuais buscam candidatos cujas forças sugiram capacidade de apresentar bons resultados em domínios específicos do trabalho. Para isso são submetidos a treinamentos que visam a aprimorar seus conhecimentos e habilidades. Organizações que utilizam essa abordagem têm alcançado elevados níveis de satisfação dos clientes internos e externos, produtividade, rendimentos, e diminuição dos níveis de turnover e de acidentes (Harter, Schmidt, \& Hayes, 2002). Entre os riscos de se adotar essa noção sobre talentos está a diminuição nos níveis de comprometimento e motivação dos profissionais, quando privados de programas que visam ao desenvolvimento de áreas nas quais eles não demonstram nível elevado de força (Martin \& Schmidt, 2010).

\section{Talento como Soma de Habilidades (ou Alto Desempenho ou Alto Potencial)}

Essa forma de conceber talento iniciou-se com o trabalho de Michaels et al. (2001). Nele os autores definiram talento de forma ampla e inespecífica como a soma das habilidades de um profissional: dons naturais, habilidades, conhecimentos, experiência, inteligência, julgamento, atitudes, caráter e motivação, além da facilidade para aprender e da disposição para buscar o desenvolvimento pessoal. Essa definição evidentemente inclui aspectos inatos, prática deliberada e forças individuais. Contudo, diferentemente das perspectivas anteriores, o entendimento de talento como produto de habilidades deriva da prática profissional. Sendo assim, carece de aprimoramento e desenvolvimento teórico e evidência empírica sistemática. Como consequência, verifica-se uma variedade de estudos desenvolvidos sob essa perspectiva, mas ainda pouco consenso sobre como definir e caracterizar talento.

Tendo como base as proposições iniciais de Michaels et al. (2001) diversos pesquisadores buscaram compreender melhor a natureza dos altos talentos e seu impacto para as organizações. De maneira geral, eles salientaram que os altos talentos compõem um grupo privilegiado de profissionais que é responsável por grande parte da receita, do lucro e do sucesso geral das organizações onde trabalham (O'Boyle \& Aguinis, 2012); que apresentam resultados superiores aos de seus pares, mesmo em contextos complexos e desafiadores (Martin \& Schmidt, 2010); e que são destacados como o mais alto capital humano em suas áreas de atuação, os $10 \%$ a $20 \%$ "melhores e mais brilhantes," ou os profissionais de "Nível A" (Michaels et al., 2010). Por essas razões, tais trabalhadores recebem ofertas salariais que ultrapassam a média praticada para seus cargos e que estão relacionadas a níveis de mobilidade mais elevados do que o de outros profissionais (Martin \& Schmidt, 2010). Esse conjunto de características levou alguns autores a afirmar que pessoas assim são tão raras que não há altos talentos suficientes para suprir a demanda do mercado (Allen, Bryant, \& Vardaman, 2010), sendo necessário, portanto, "lutar" por eles. 
Outros pesquisadores apresentaram tentativas de caracterizar talento e apontar aspectos que facilitariam ou dificultariam sua expressão (Pepermans et al., 2003). Para eles, o alto talento caracterizaria uma categoria de profissionais que apresenta qualidades (ou competências) tão peculiares que poderiam transformá-los nos futuros líderes das organizações onde trabalham. De acordo com Pepermans et al. (2003), tais competências são: trabalho em equipe; motivação para alcançar melhores desempenhos; delegação de tarefas; liderança, persuasão, julgamento; ousadia; capacidade de assumir riscos; criatividade; identificação com a organização; flexibilidade; visão; integridade; consciência da existência de diferenças culturais; capacidade de oferecer feedback adequado. Entre as variáveis mediadoras que facilitam a expressão do talento, estariam: a motivação, o autoconceito, as estratégias de coping e outros traços de personalidade (Heller \& Perleth, 2008).

Há também aqueles que sugeriram que o talento requer uma visão holística e propuseram equações e modelos para explicá-lo (Silzer \& Church, 2009; Ulrich, 2006). Um exemplo é a equação: talento $=$ competência + comprometimento + contribuição (Ulrich, 2006). Nessa equação, a competência se refere ao conhecimento, às habilidades e aos valores que são e serão demandados do profissional no presente $\mathrm{e}$ no futuro. O comprometimento está relacionado ao esforço e ao tempo despendido para realizar atividades propostas pela organização visando ao sucesso. Por fim, a contribuição diz respeito ao quão relevante uma atividade de trabalho é na perspectiva do profissional e da organização. Outro exemplo é a equação: talento = aspectos fundamentais + aspectos de crescimento + aspectos de carreira (Silzer \& Church, 2009). Nela, os aspectos fundamentais se referem a atributos naturais e estáveis relacionados à cognição e à personalidade. Os aspectos de crescimento se referem à habilidade para aprender e à motivação. Finalmente as dimensões de carreira dizem respeito à habilidade de liderança, desempenho, conhecimento, e valores (Silzer \& Church, 2009).

Sob essa noção de soma de habilidades, observa-se uma grande quantidade de publicações que enfatizam a importância de avaliar e identi- ficar altos talentos. De fato, é possível encontrar diversos outros estudos, além dos citados, que fazem tentativas de definir o que é o talento ou a que ele se refere. No entanto, raras são as publicações que apresentam sugestões e indicações sobre como acessar esse fenômeno (McDonnell, 2011). Na ausência de instrumentos específicos para esse fim, a principal estratégia para acessar talento enquanto soma de habilidades é o emprego das medidas de avaliação de desempenho realizadas pelas organizações (Dries \& Pepermans, 2008). Tais avaliações permitem discriminar diferentes níveis de talento e adotam uma perspectiva de metacompetências para explorar as características de cada nível. Alguns exemplos são: a inteligência emocional (Dries \& Pepermans, 2008) e o desenvolvimento da identidade (Hall, 2002).

As organizações que veem o talento sob a visão da soma das habilidades tendem a entender que os altos talentos são os profissionais que consistentemente e significativamente apresentam desempenho superior ao de seus pares em diversos contextos. Além disso, compreendem que os comportamentos exibidos pelos profissionais talentosos refletem, de maneira exemplar, a cultura e os valores da organização onde trabalham (Ready et al., 2010). Por esses aspectos, mostram uma grande capacidade de crescer e ter sucesso na carreira com seus empregadores de forma mais efetiva e mais rápida que seus pares (Ready et al., 2010).

$\mathrm{O}$ maior risco que as organizações correm ao adotar a perspectiva de talento como soma de habilidades é entender o fenômeno como tão singular que se torne impossível encontrar no mercado ou dentro da própria organização profissionais altamente talentosos. Um possível resultado dessa visão é a manutenção da "guerra por talentos" e da crença de que não há profissionais suficientes para atender a demanda das organizações.

\section{Caracterização do Estudo}

Este estudo teve como um de seus objetivos desenvolver um instrumento para aferir o talento em contexto organizacional. Para tanto, partiu-se da noção de talento de Ready et al. (2010) 
que compreende o talento como o produto de habilidades. Em princípio, essa perspectiva não negaria as demais concepções sobre o talento e permitiria integrar a noção de habilidades pessoais ao processo de execução de tarefas relacionadas ao trabalho. Assim, em uma tentativa de estender o escopo da avaliação a diversos contextos organizacionais, definiu-se o construto talento como: conjunto de habilidades inter-relacionadas apresentadas por profissionais especificamente na execução do seu trabalho e que contribuem positivamente para os objetivos da organização. Essas habilidades devem compreender três dimensões que dizem respeito à qualidade, à velocidade e à autonomia no desempenho das atividades.

A dimensão qualidade refere-se à realização das atividades de trabalho com excelência, ao alto nível de qualidade sobre o produto do trabalho. A velocidade diz respeito à agilidade com que o trabalho é executado, à rapidez em concluir as tarefas no trabalho. Por fim, a autonomia está relacionada à capacidade de desenvolver as próprias atividades de trabalho ou resolver problemas do trabalho sem necessitar da ajuda de colegas e supervisores. Decorrente dessa noção de talento, pessoas que apresentem elevados níveis em todas as dimensões são capazes de desenvolver suas atividades de trabalho com autonomia e excelência dentro do tempo esperado ou em menos tempo do que é esperado. Pessoas com baixos níveis em todas as dimensões apresentam dificuldades para desenvolver as próprias atividades de trabalho com autonomia, apresentam resultados de baixa qualidade e demoram muito para apresentar os resultados de seu trabalho

\section{Método}

\section{Participantes}

Participaram deste estudo 308 profissionais ativos no mercado de trabalho, $61 \%$ eram homens, média de idade de 35,1 anos $(D P=8,79)$. Do total de participantes, $46,4 \%$ tinham concluído uma pós-graduação, 35,7\% tinham ensino superior completo e $17,9 \%$ tinham o ensino médio completo. A maioria deles, $87,4 \%$, estava empregada em empresas privadas e $12,6 \%$ em instituições públicas. Os salários mensais recebidos variaram de $\mathrm{R} \$ 600,00$ a $\mathrm{R} \$ 25.000,00$ $(M=5.961,4 ; D P=4.291,0)$. A média de aumentos salariais recebidos nos últimos três anos foi de 3,32 $(D P=1,64)$; e a média de promoções recebidas também nos últimos três anos foi de 1,84 $(D P=0,97)$. Os participantes declararam trabalhar em média 45,5 horas por semana $(D P=10,2)$.

\section{Instrumentos}

Utilizou-se um questionário informatizado hospedado em um endereço na internet com questões sociodemográficas como sexo, idade e escolaridade, e perguntas que abrangiam tópicos relacionados à empresa, à personalidade e à avaliação de talento.

Empresa. Questões como: qual o salário mensal; quantas horas trabalha por semana; trabalha em empresa privada ou pública; quantos aumentos recebeu nos últimos três anos; quantas promoções recebeu nos últimos três anos; se recebeu oferta de trabalho de outras organizações nos últimos 12 meses.

Personalidade. Utilizou-se um instrumento para aferir a personalidade a partir do modelo dos cinco grandes fatores: Marcadores Reduzidos (Hauck, Machado, Teixeira, \& Bandeira, 2012). O instrumento é composto por 25 adjetivos seguidos de uma escala de cinco pontos em que os participantes devem responder o quanto concordam que os adjetivos os descrevem adequadamente. Cada conjunto de cinco adjetivos diz respeito a um grande fator da personalidade, tais quais: socialização, extroversão, neuroticismo, conscienciosidade e abertura. Os coeficientes alfa do instrumento em sua versão original variaram de 0,61 a 0,83 .

Avaliação de Talento. Utilizou-se um conjunto de 20 itens em formato de frases afirmativas, seguidos de uma escala de cinco pontos para que os participantes respondessem o quanto concordavam com elas. Os itens abrangem três dimensões do talento que se referem à qualidade na realização do trabalho, oito itens; a velocidade na realização do trabalho, seis itens; e a autonomia na realização do trabalho, seis itens. Esse instrumento foi desenvolvido especificamente para este estudo. Os procedimentos de elabora- 
ção e evidências de validade e precisão são apresentados ao longo do manuscrito.

\section{Procedimentos}

Elaboração dos Itens. A partir da definição do construto, concebido como tridimensional, elaboraram-se trinta e quatro afirmativas relacionadas ao contexto organizacional, que pudessem representar o talento nas suas três dimensões. Foram elaborados tantos itens quanto os pesquisadores conseguiram. Para a qualidade elaboraram-se 14 itens, para a velocidade, 10 itens, e para a dimensão autonomia 10 itens. Em seguida, encaminharam-se os itens, juntamente com uma descrição de cada uma das três dimensões, a quatro juízes alheios a esta pesquisa. Os juízes, estudantes de graduação e pós-graduação em psicologia, foram convidados a enquadrar cada afirmativa em uma das dimensões descritas. Além disso, solicitou-se que eles avaliassem o quanto consideravam as afirmativas compreensíveis. De maneira independente, os juízes enquadraram as afirmativas nas dimensões propostas, deram sugestões de reescrita e eliminação de itens com conteúdo repetido. A partir desse procedimento retiveram-se para passos subsequentes da pesquisa 20 itens que foram consensualmente enquadrados nas suas respectivas categorias e que não apresentaram problemas de compreensão.

Coleta de Dados. Os participantes foram recrutados através de redes sociais e profissionais. Para aqueles que apresentavam o perfil esperado foram enviadas mensagens pessoais com convites para participar da pesquisa. Nas mensagens havia uma breve descrição do estudo e o endereço eletrônico do questionário. Ao entrar no site, os participantes podiam ler explicações sobre a pesquisa, sobre como responder ao questionário, bem como, o termo de consentimento livre e esclarecido. Aqueles que consentiam em participar tinham acesso ao questionário.

O questionário em formulário eletrônico foi configurado para impedir a existência de casos omissos para os itens das escalas utilizadas. Desenvolveu-se esse estudo seguindo-se todos os preceitos éticos de pesquisas com seres humanos, conforme resolução 196/96 do Conselho Nacional de Saúde.

\section{Resultados}

Em um primeiro momento, testou-se de modo exploratório a estrutura do conjunto de itens elaborados para aferir o talento, a partir de uma análise de componentes principais com método de rotação oblimin. Utilizou-se esse método considerando-se que a definição do construto sugere dimensões correlacionadas, além disso, tal método não impede a emergência de fatores oblíquos (Pasquali, 2005). Verificou-se adequação dos dados à fatorização, $\mathrm{KMO}=0,90$ e teste de esfericidade de Bartlett: $\chi^{2}(190, N=308)=2252,9$; $p<0,001$. Constatou-se haver três dimensões com eigenvalue $>1$, as quais explicaram $52,6 \%$ da variância dos dados. O screeplot mostrou a emergência de três evidentes dimensões, ainda, uma análise paralela de eigenvalues aleatórios permitiu constatar que o último eigenvalue observado maior que o simulado foi o da dimensão três (dimensão quatro: eigenvalue observado $=0,91$ e eigenvalue simulado $=1,31$ ). Tendo-se em vista os critérios de retenção de fatores citados acima, respectivamente: de Kaiser (1960), de Cattell (1966) e de Horn (1965), e sobretudo a definição que fundamenta o construto, considerou-se adequada a extração de três dimensões para o instrumento.

Os itens, suas respectivas cargas componenciais em cada dimensão, comunalidades e outras propriedades psicométricas do instrumento podem ser vistos na Tabela 1. Verificou-se que o agrupamento empírico dos itens foi condizente com o julgamento dos juízes na etapa anterior de elaboração do instrumento. A dimensão 1 (D1) agrupou os oito itens referentes à qualidade do trabalho, na dimensão 2 (D2) carregaram os seis itens relacionados à velocidade de execução do trabalho, e a dimensão 3 (D3) foi composta pelos seis itens referentes à autonomia na realização do trabalho. Como esperado, todas as dimensões mostraram-se correlacionadas, tal que entre D1 e D2 obteve-se $r=0,33$; entre D1 e D3, $r=0,56$; e entre $\mathrm{D} 2$ e $\mathrm{D} 3, r=0,22$. No que diz respeito à fidedignidade, os coeficientes alfa (Cronbach, 1951) de cada dimensão foram todos superiores a 0,80 , indicando satisfatórios índices de precisão do instrumento (Nunnally, 1967). 
Tabela 1

Cargas Componenciais e Comunalidades dos Itens a Partir de uma Análise de Componentes Principais com Rotação Oblimin

\begin{tabular}{|c|c|c|c|c|}
\hline Itens & D1 & D2 & D3 & $h^{2}$ \\
\hline $\begin{array}{l}\text { 7. Com frequência as pessoas ficam admiradas com a qualidade } \\
\text { do meu trabalho. }\end{array}$ & $\mathbf{0 , 8 3}$ & $-0,02$ & 0,09 & 0,64 \\
\hline 10. Posso ser considerado o número 1 no trabalho que faço. & $\mathbf{0 , 7 3}$ & 0,03 & 0,07 & 0,47 \\
\hline $\begin{array}{l}\text { 18. Meus superiores consideram meu desempenho melhor do que } \\
\text { a média. }\end{array}$ & 0,67 & $-0,08$ & $-0,07$ & 0,54 \\
\hline 13. Meus colegas de trabalho dizem que eu sou bom no que faço. & 0,66 & $-0,08$ & $-0,02$ & 0,49 \\
\hline $\begin{array}{l}\text { 12. Sinto que tenho algum talento especial que me faz realizar bem meu } \\
\text { trabalho. }\end{array}$ & 0,66 & 0,15 & 0,002 & 0,39 \\
\hline $\begin{array}{l}\text { 20. Quando as pessoas precisam que alguma coisa seja feita com perfeição no } \\
\text { trabalho buscam a minha ajuda. }\end{array}$ & 0,62 & $-0,05$ & $-0,06$ & 0,45 \\
\hline $\begin{array}{l}\text { 1. Geralmente me destaco em minha equipe pela boa qualidade } \\
\text { do meu trabalho. }\end{array}$ & $\mathbf{0 , 6 0}$ & $-0,14$ & $-0,08$ & 0,48 \\
\hline $\begin{array}{l}\text { 4. Costumo ouvir que sou melhor do que outras pessoas que fazem atividades } \\
\text { semelhantes à minha. }\end{array}$ & $\mathbf{0 , 5 8}$ & $-0,02$ & $-0,14$ & 0,44 \\
\hline 8. Costumo atrasar para concluir minhas atividades de trabalho. & $-0,10$ & 0,76 & $-0,06$ & 0,62 \\
\hline $\begin{array}{l}\text { 16. Acho que o que falta para que eu seja o número } 1 \text { no que eu faço } \\
\text { é concluir minhas atividades mais rapidamente. }\end{array}$ & 0,02 & $\mathbf{0 , 7 4}$ & $-0,16$ & 0,52 \\
\hline 6. Costumo demorar mais do que outras pessoas para fazer o meu trabalho. & 0,04 & $\mathbf{0 , 7 3}$ & 0,13 & 0,57 \\
\hline $\begin{array}{l}\text { 11. Costumo concluir minhas atividades de trabalho no último } \\
\text { minuto possível. }\end{array}$ & 0,05 & $\mathbf{0 , 7 2}$ & $-0,05$ & 0,50 \\
\hline 2. Costumo ouvir que demoro para fazer minhas atividades de trabalho. & $-0,09$ & $\mathbf{0 , 7 0}$ & 0,07 & 0,56 \\
\hline $\begin{array}{l}\text { 14. Levo mais tempo que as outras pessoas normalmente levam para fazer } \\
\text { meu trabalho. }\end{array}$ & $-0,04$ & 0,68 & 0,17 & 0,56 \\
\hline 15. Geralmente consigo fazer meu trabalho sozinho. & $-0,02$ & 0,01 & $-0,78$ & 0,60 \\
\hline 5. Geralmente consigo fazer meu trabalho sem precisar de ajuda & 0,08 & $-0,07$ & $-0,72$ & 0,60 \\
\hline $\begin{array}{l}\text { 17. Quando não sei como fazer alguma atividade de trabalho, } \\
\text { consigo descobrir sozinho. }\end{array}$ & $-0,01$ & 0,12 & $-0,72$ & 0,49 \\
\hline 9. Soluciono problemas de trabalho sem ajuda de outras pessoas. & 0,10 & 0,06 & $-0,70$ & 0,55 \\
\hline 19. Consigo perceber sozinho como fazer um trabalho melhor. & $-0,08$ & $-0,10$ & $-0,69$ & 0,46 \\
\hline $\begin{array}{l}\text { 3. Frequentemente sou capaz de encontrar sozinho soluções para problemas } \\
\text { de trabalho. }\end{array}$ & 0,24 & $-0,07$ & $-0,62$ & 0,62 \\
\hline Eigenvalues & 6,34 & 2,62 & 1,56 & \\
\hline \% Total de Variância Explicada & & 52,6 & & \\
\hline Média & 3,75 & 1,91 & 3,68 & \\
\hline Desvio-Padrão & 0,65 & 0,71 & 0,72 & \\
\hline Coeficiente Alfa (Cronbach, 1951) & 0,84 & 0,82 & 0,83 & \\
\hline
\end{tabular}

Nota. D1-qualidade do trabalho; D2-velocidade na execução do trabalho (itens devem ser invertidos); D3-autonomia na realização do trabalho. Cargas componenciais $>0,30$ estão em negrito. 
Levando-se em conta a existência de um modelo definido sustentando a existência de três fatores para o construto, testou-se a estrutura do instrumento também de modo confirmatório, a partir de uma análise fatorial confirmatória. Adicionalmente, comparou-se o modelo de três fatores com um modelo hipotético de fator único. Para ambos os modelos partiu-se da matriz de covariância dos dados para a estimação dos parâmetros e optou-se pelo algoritmo Maximum Likelihood. Utilizou-se o software AMOS 16.0 para essas análises. No modelo de três fatores especificou-se uma estrutura com os três fatores correlacionados, cada um explicado por suas respectivas observáveis, como podem ser vistos na Tabela 1: fator 1 - oito itens referentes à qualidade, a saber: 7, 10, 18, 13, 12, 20, 1 e 4; fator 2 - seis itens referentes à velocidade, a saber: 8 , $16,6,11,2$ e 14; fator 3 - seis itens referentes à autonomia, a saber: $15,5,17,9,19$ e 3. Para o modelo de fator único especificou-se um fator explicado por todos os itens. O ajuste dos dados aos modelos testados foi avaliado a partir dos seguintes índices, em acordo com o que tem sido sugerido pela literatura especializada (Byrne, 2009; Jackson, Gillaspy, \& Purc-Stephenson, 2009): razão entre qui-quadrado e graus de liberdade $\left(\chi^{2} / g l\right)$; Goodness-of-Fit Index (GFI); Adjusted Goodness-of-Fit Index (AGFI); Normed Fit Index (NFI); Tucker-Lewis Index (TLI); Comparative Fit Index (CFI); Root Mean Square Error of Aproximation (RMSEA) e Consistent Akaike Information Criterion (CAIC).

A Tabela 2 mostra os índices de ajuste obtidos com a análise fatorial confirmatória para os dois modelos testados. Observou-se que a estrutura de três fatores apresenta melhor ajuste comparado ao modelo de fator único (CAIC três fatores $<$ CAIC um fator). Ainda, os índices obtidos para o modelo de três fatores, quando tomados em conjunto, sugerem adequação dos dados à estrutura do instrumento, considerando-se que $\chi^{2} / g l<2$; RMSEA $<0,06$; CFI, TLI e GFI $>0,90$ e NFI e AGFI próximos a 0,90 (Byrne, 2009; Marsh, Hau, \& Wen, 2004).

Tabela 2

\section{Índices dos Modelos Testados a Partir de Análise Fatorial Confirmatória}

\begin{tabular}{lcc}
\hline & \multicolumn{2}{c}{ Modelos } \\
\cline { 2 - 3 } & Três Fatores & Um Fator \\
\hline$\chi^{2}$ & 283,3 & 880,4 \\
$g l$ & 167 & 170 \\
$P$ & $<0,001$ & $<0,001$ \\
$\chi^{2} / g l$ & 1,70 & 5,18 \\
GFI & 0,92 & 0,70 \\
AGFI & 0,89 & 0,63 \\
NFI & 0,88 & 0,62 \\
TLI & 0,94 & 0,62 \\
CFI & 0,94 & 0,66 \\
RMESA & 0,048 & 0,12 \\
IC 90\% RMESA & $0,038-0,057$ & $0,11-0,12$ \\
CAIC & 572,7 & 1149,6 \\
\hline
\end{tabular}

Nota. $\chi^{2}$ - qui-quadrado; $g l$ - graus de liberdade; $\chi^{2} / g l$ - razão qui-quadrado por graus de liberdade; GFI - Goodness-of-Fit Index; AGFI - Adjusted Goodness-of-Fit Index; NFI - Normed Fit Index; TLI - Tucker-Lewis Index; CFI - Comparative Fit Index; RMSEA - Root Mean Square Error of Aproximation; IC 90\% RMESA - Intervalo de confiança de 90\%; CAIC - Consistent Akaike Information Criterion. 


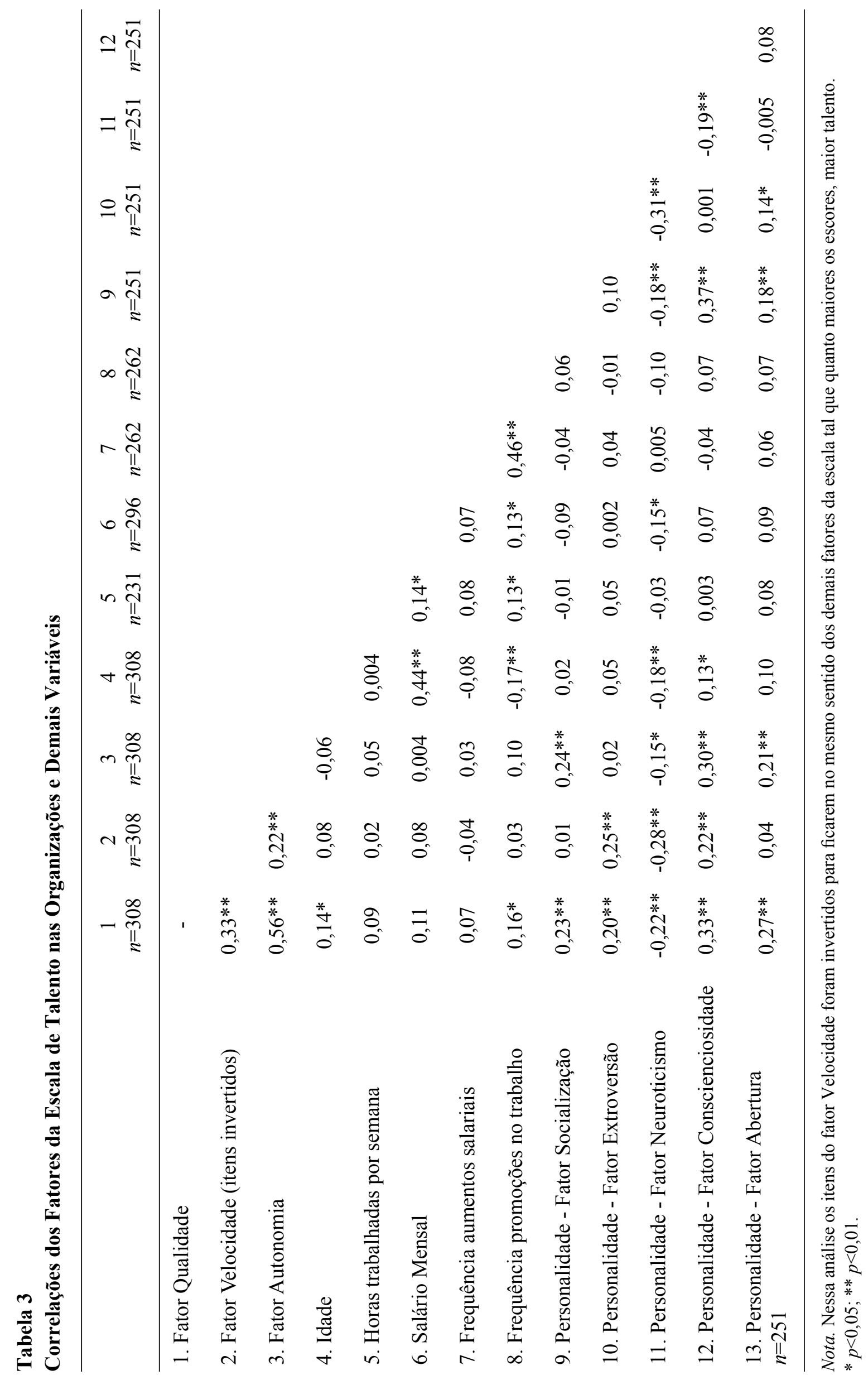


Além de testes da adequação da estrutura do instrumento, examinaram-se relações entre os fatores do Talento e construtos e variáveis observáveis teoricamente correlatas. Para essa análise e todas as subsequentes inverteram-se os itens do fator Velocidade da escala de Talento, a fim de deixar esse fator no mesmo sentido que os outros dois da escala, tal que quanto maiores os escores, maior percepção de talento. Os resultados da análise de correlação de Pearson entre as variáveis investigadas podem ser vistos na Tabela 3. Destacam-se as correlações positivas de todos os fatores do Talento e o fator de personalidade Conscienciosidade e negativas com o fator Neuroticismo.

Por fim, testaram-se diferenças no Talento para as variáveis sociodemográficas como sexo, escolaridade e tipo de empresa e para a seguinte questão critério: recebeu oferta de emprego de outras empresas nos últimos 12 meses? Considerando-se o construto multidimensional realizaram-se MANCOVAs para cada variável investigada. Nessas análises incluiu-se a idade como covariável, tendo em vista sua correlação com uma dimensão do construto. Não foram verificadas diferenças no talento percebido entre homens e mulheres, $\Lambda$ de Wilks $=0,99 ; F(3,303)=1,02$; $p=0,38 ; \eta^{2}=0,01$; também não houve diferenças entre profissionais que tinham concluído apenas ensino médio, aqueles que concluíram ensino superior e os que concluíram pós-graduação, $\Lambda$ de Wilks=0,98; $F(6,604)=1,07 ; p=0,38$; $\eta^{2}=0,01$; tampouco se encontraram diferenças no Talento entre profissionais que trabalhava em empresa pública ou privada, $\Lambda$ de Wilks $=0,99$; $F(3,264)=0,54 ; p=0,65 ; \eta^{2}=0,01$.

Contudo, entre os profissionais que receberam oferta de emprego de outra empresa nos últimos 12 meses $(n=175)$ e aqueles que não receberam qualquer oferta de emprego nos últimos 12 meses $(n=87)$, foi verificada diferença no Talento, $\Lambda$ de Wilks $=0,97 ; F(3,257)=2,88 ; p=0,037$; $\eta^{2}=0,03$. As análises univariadas realizadas em seguida, permitiram constatar que aqueles que receberam oferta de emprego $(M=3,81$; $D P=0,62)$ apresentaram maior média no fator Qualidade quando comparados aos que não receberam oferta de emprego $(M=3,63 ; D P=0,68)$, $F(1,259)=4,82 ; p=0,040 ; d=0,26$.

\section{Discussão}

Este estudo teve como objetivo desenvolver e apresentar evidências de validade de uma medida para aferir o talento. As análises exploratórias e confirmatórias evidenciaram a adequação da estrutura tridimensional e inter-relacionada do instrumento, em conformidade com a definição do construto. Os índices de ajuste dos dados ao modelo de três fatores, tomados em conjunto, satisfazem os critérios mínimos de adequação (Byrne, 2009; Marsh et al., 2004). Também se obteve índices satisfatórios de precisão para o instrumento (Nunnally, 1967). Adicionalmente, testaram-se relações entre os fatores da escala de talento com variáveis destacadas na literatura como correlatas: sexo (Beechler \& Woodward, 2009; Hewlett \& Rashid, 2011), idade (Beechler \& Woodward, 2009), escolaridade (Ericsson, 2008), tipo de empresa e o recebimento ou não de oferta de emprego nos últimos 12 meses (Martin \& Schmidt, 2010) e traços de personalidade (Garcia-Santos et al., 2012; Heller \& Perleth, 2008; Silzer \& Church, 2009).

Não se verificou diferenças no talento entre homens e mulheres, o que está de acordo com a similaridade de características entre os sexos na realização de tarefas nos mais diversos âmbitos (Hyde, 2005). Também não houve diferenças entre aqueles que trabalhavam em instituição pública e privada, o que contribui para a desconstrução do preconceito frente a funcionários públicos brasileiros, e ressalta a distribuição de pessoas talentosas em diversos âmbitos da sociedade. Ainda, não foram verificadas diferenças no talento entres os níveis de escolaridade, fortalecendo a noção integrativa (indivíduo e execução da tarefa) do construto elaborado. Esse resultado sugere que o talento não está associado unicamente ao nível de educação formal, ideia que vai ao encontro das proposições de Ready et al. (2010). Segundo esses autores, os altos talentos não seriam caracterizados como aprendizes extremamente dedicados e incansáveis, mas sim indivíduos motivados a explorar novas ideias e capazes de transformá-las em ações frutíferas para si mesmos e para as organizações.

Verificou-se ainda uma correlação positiva, ainda que fraca, entre o fator Qualidade de 
talento e a idade dos participantes. Essa relação vai ao encontro das proposições da perspectiva de talento enquanto prática deliberada (Ericsson, 2004; 2008; Ericsson \& Lehman, 1996; Ericsson et al., 2007). Uma vez que os profissionais com idades superiores provavelmente acumularam mais horas de prática na execução de atividades similares ou idênticas a sua atual ocupação, espera-se que eles também apresentem maior qualidade na execução de seu trabalho. Ainda que essa relação entre talento e idade seja esperada, é comum encontrar organizações que depositam nos profissionais mais jovens suas esperanças de encontrar os altos talentos e futuros líderes (Martin \& Schmidt, 2010).

No que diz respeito ao histórico profissional, não foram encontradas relações entre talento e salário mensal, horas trabalhadas por semana e aumentos recebidos nos últimos três anos. Acredita-se que a existência de acordos e leis trabalhistas no Brasil que visam a aumentos salariais anuais para categorias profissionais, equiparações salariais para cargos semelhantes e regulamentações sobre carga horária máxima de trabalho contribuíram para a impossibilidade de se encontrarem relações do talento com esses critérios neste estudo. Por outro lado, o número de promoções recebidas, que pode ser considerada uma variável mais maleável dentro das organizações, comparada às supracitadas que têm regulamentações, mostrou-se correlacionada positivamente com o fator Qualidade do talento. Embora o valor da correlação tenha sido baixo, esse resultado sugere que quanto maior o nível de qualidade do trabalho maior o número de promoções recebidas nos últimos três anos, tal como defendem também outros autores (DeVaro, Ghosh, \& Zoghi, 2007).

No que diz respeito à personalidade, eram esperadas correlações positivas de talento com o fator Conscienciosidade, tal como foi observado em estudos sobre desempenho profissional (Barrick \& Mount, 1991). Esse fator, comumente chamado no Brasil de Realização, está relacionado ao grau de organização, persistência, controle e motivação para atingir metas (Filho et al., 2012; Nunes, Hutz, \& Nunes, 2010). Pessoas que têm altos escores nesse fator tendem a ser organizadas, confiáveis, trabalhadoras, decididas, pontuais, escrupulosas, ambiciosas e perseverantes (Nunes et al., 2010). Os resultados indicaram relações desse fator de personalidade com todas as dimensões de talento, o que reforça a validade do instrumento.

Outro fator de personalidade que apresentou correlações com as três dimensões do talento foi o Neuroticismo, nesse caso correlações negativas. Neuroticismo diz respeito ao ajustamento e à instabilidade emocional, representa a maneira como as pessoas lidam com um desconforto psicológico (Hauck et al., 2012; Nunes et al., 2010). Níveis elevados nesse fator sugerem pessoas que vivenciam com mais intensidade sofrimentos emocionais, preocupações, irritação, melancolia, vergonha; descrevem pessoas com tendência a hostilidade, ansiedade, depressão, autocrítica e baixa tolerância à frustração (Nunes et al., 2010). Características como essas, independentemente da área de atuação, tendem a inibir a conclusão das atividades de trabalho; consequentemente, relações negativas com todas as dimensões do talento vão ao encontro dos achados sobre o desempenho profissional (Barrick \& Mount, 1991; Garcia-Santos et al., 2012).

Os outros fatores de personalidade também apresentaram correlações com pelo menos uma dimensão do talento, com destaque para a dimensão Qualidade que se correlacionou com todos os fatores. As correlações com todas as variáveis, à exceção do Neuroticismo, foram positivas. $\mathrm{O}$ fator Socialização refere-se a características de relacionamento interpessoal direcionadas aos outros. Caracteriza pessoas corteses, flexíveis, confiáveis, cooperativas, e tolerantes (Hauck et al., 2012; Nunes et al., 2010). O fator Extroversão diz respeito à quantidade e à intensidade de interações sociais, sinaliza indivíduos agregadores, falantes e ativos (Hauck et al., 2012; Nunes et al., 2010). Ambas as dimensões são características de profissionais capazes de estabelecer boas relações interpessoais no trabalho, e de desenvolver atividades laborais em equipe, características que poderiam contribuir para a melhor qualidade do trabalho realizado (Barrick \& Mount, 1991). Por sua vez, a Abertura para experiências se caracteriza por ser um 
fator norteador da busca e valorização de comportamentos exploratórios e inéditos, descreve pessoas imaginativas, curiosas, originais, cultas, inteligentes, sensíveis à arte e liberais (Hauck et al., 2012; Nunes et al., 2010). Segundo Barrick e Mount (1991) profissionais que têm alta abertura à experiência tendem a buscar maior aprimoramento através de treinamentos, por exemplo, o que resultaria em maior qualidade do trabalho desempenhado. Esses resultados das relações entre fatores de personalidade e desempenho profissional foram apontados na meta-análise de Barrick e Mount (1991) e fortalecem a noção de inter-relação de características pessoais com características da execução do trabalho, tal como proposto para neste estudo.

Por fim, o resultado de maiores níveis de talento entre aqueles que receberam convite de trabalho de outras organizações nos últimos 12 meses era esperado e está em acordo com a argumentação de DeVaro et al. (2007) sobre competição no mercado de trabalho. Segundo DeVaro et al. (2007) profissionais talentosos cuja qualidade do trabalho é reconhecida, por exemplo por meio de aumentos salarias e promoções, são colocados em evidência e passam a ser cobiçados por outras organizações. Sobre esse ponto considera-se importante ressaltar que o resultado da MANCOVA, embora significativo, foi bastante modesto. Os resultados dos testes univariados revelaram que apenas o fator Qualidade mostrou-se diferente entre os dois grupos de participantes. Portanto, novos estudos conduzidos com a escala devem estar atentos a isso.

Entre as limitações deste estudo, considera-se pertinente ressaltar o fato de a amostra ter sido recrutada exclusivamente via internet. Se por um lado esse recrutamento permitiu acesso a profissionais ativos, provenientes de diferentes organizações, por outro ele impossibilitou o acesso a dados dos participantes gerados em suas organizações, como resultados de avaliação de desempenho. Informações desse tipo poderiam ser utilizadas na busca de evidências de validade para o instrumento, e também para revelar relações causais importantes sobre o fenômeno. Outra limitação que merece destaque, relacionada a instrumentos de autorrelato, é o efeito de leniência (Heidemer \& Moser, 2009). Esse efeito preconiza que quando profissionais são solicitados a emitir uma avaliação sobre si, tendem a fazer uma avaliação mais positiva do que aquela feita por supervisores ou pares. Acredita-se que futuros estudos utilizando a Escala de Talento nas Organizações seriam promissores se obtivessem também dados externos provenientes diretamente das organizações ou de colegas.

Apesar das limitações, considera-se que o instrumento elaborado traz contribuições importantes para a academia e para a prática profissional. Em termos práticos a escala pode ser empregada em processos de avaliações de desempenho, como forma de ter acesso à perspectiva dos profissionais sobre sua qualidade, velocidade e autonomia na execução do trabalho. Isso permitiria ampliar o conhecimento sobre como o profissional percebe seu trabalho e facilitaria a elaboração de estratégias para alinhar as expectativas organizacionais com a visão sobre o talento de seus trabalhadores. Nesse mesmo sentido, o instrumento poderia ser empregado para estabelecer e monitorar o alcance de metas em planos de desenvolvimento individual, no que diz respeito às três dimensões avaliadas, visando um melhor desempenho do profissional no trabalho.

\section{Conclusão}

Revisou-se a literatura sobre talento e elaborou-se uma definição de construto que compreende o talento a partir de três dimensões correlacionadas referentes à qualidade, velocidade e autonomia na execução do trabalho em organizações. A definição ainda entende o talento como produto de uma interação de características pessoais com a atividade de trabalho. Elaborou-se um instrumento para aferir o construto e encontraram-se evidências que sustentam sua validade e precisão. Considera-se pertinente que novos estudos sejam desenvolvidos com vistas tanto ao aperfeiçoamento do instrumento como à busca por relações do talento com outras variáveis. 


\section{Referências}

Aguinis, H., Gottfredson, R. K., \& Joo, H. (2012). Using performance management to win the talent war. Business Horizons, 55, 609-616. doi: 10.1016/j.bushor.2012.05.007

Allen, D. G., Bryant, P. C., \& Vardaman, J. M. (2010). Retaining talent: Replacing Misconceptions with evidence-based strategies. Academy of Management Perspectives, 24(2), 48-64.

Barrick, M. R., \& Mount, M. K. (1991). The Big Five personality dimensions and job performance: A meta-analysis. Personnel Psychology, 44(1), 1-26. doi: 10.1111/j.1744-6570.1991.tb00688.x

Beechler, S., \& Woodward, I. C. (2009). The global war for talent. Journal of International Management, 15(3), 273-285. doi: 10.1016/j.intman.2009.01.002

Bloom, B. S. (1985). Generalizations about talent development. In B. S. Bloom (Ed.), Developing talent in young people (pp. 507-549). New York: Ballantine Books.

Byrne, B. M. (2009). Structural equation modeling with AMOS: Basic concepts, applications, and programming ( $2^{\text {nd }}$ ed.). New York: Routledge.

Cattell, R. B. (1966). The scree test for the number of factors. Multivariate Behavioral Research, 1, 245-276. doi: 10.1207/s15327906mbr0102_10

Chambers, E., Foulon, M., Handfield-Jones, H., Hankin, S., \& Michaels, E. (1998). The war for talent. The McKinsey Quarterly, 3, 44-57.

Coffman, C., \& Gonzalez-Molina, G. (2002). Follow this path: How the world's greatest organizations drive growth by unleashing human potential. New York: Warner Books.

Cronbach, L. (1951). Coefficient alpha and the internal structure of tests. Psychometrika, 16(3), 297 334. doi: $10.4135 / 9781412961288$

DeBruin, A. B. H., Rikers, R. M. J. P., \& Schmidt, H. G. (2007). The influence of achievement motivation and chess-specific motivation on deliberate practice. Sport Psychology, 29, 562-583.

DeGroot, A. D. (1965). Thought and choice in chess. The Hague, Netherlands: Mouton.

DeVaro, J., Ghosh, S., \& Zoghi, C. (2007). Job characteristics and labor Market discrimination in promotions: New theory and empirical evidence. East Bay, CA: Mimeo.
Dries, N., \& Pepermans, R. (2008). "Real" highpotential careers: An empirical study into the perspectives of organisations and high potentials. Personnel Review, 37(1), 85-108. doi: $10.1108 / 00483480810839987$

Ericsson, K. A. (2004). Deliberate practice and the acquisition and maintenance of expert performance in medicine and related domains. $A c a-$ demic Medicine, 79(10), 70-81.

Ericsson, K. A. (2008). Deliberate practice and acquisition of expert performance: A general overview. Academic Emergency Medicine, 15(11), 988994. doi: 10.1111/j.1553-2712.2008.00227.x

Ericsson, K. A., \& Charnes, N. (1994). Expert performance its structure and acquisition. American Psychologist, 49(8), 725-747. doi: 10.1037/0003-066X.49.8.725

Ericsson, K. A., \& Lehmann, A. C. (1996). Expert and exceptional performance: Evidence of maximal adaptation to task. Annual Review of Psychology, 47, 273-305. doi: 10.1146/annurev. psych.47.1.273

Ericsson, K. A., Roring, R. W., \& Nandagopal, K. (2007). Giftedness and evidence for reproducibly superior performance: An account based on the expert performance framework. High Ability Studies, 18(1), 3-56. doi: 10.1080/13598130701350593

Ericsson, K. A., \& Williams, A. M. (2007). Capturing naturally occurring superior performance in the laboratory: Translational research on expert performance. Journal of Experimental Psychology: Applied, 13(3), 115-123. doi: 10.1037/1076898X.13.3.115

Gagné, F. (1985). Giftedness and talent: Reexamining a reexamination of the definitions. Gifted Child Quarterly, 29(3), 103-112. doi: $10.1177 / 001698628502900302$

Gagné, F. (2009). Building gifts into talents: Detailed overview of the DMGT 2.0. In B. MacFarlane \& T. Stambaught (Eds.), Leading change in gifted education: The festschrift of Dr Joyce VanTassel-Baska (pp. 61-80). Waco, TX: Prufrock Press.

Galton, F. (1869). Hereditary Genius. London: Macmillan.

Garcia-Santos, S. C., Almeida, L. S., \& Werlang, B. S. G. (2012). Excelência Humana: A contribuição da personalidade. Paidéia (Ribeirão Preto), 22(5), 251-259. doi:10.1590/S0103$-863 \times 2012000200011$ 
Groysberg, B., Lee, L. E., \& Abrahams, R. (2010). What it takes to make star hires pay off. MIT Sloan Management Review, 51(2), 56-61.

Groysberg, B., McLean, A. N., \& Nohria, N. (2006). Are leaders portable? Harvard Business Review, $84,92-100$.

Hall, D. T. (2002). Careers in and out of organizations. Thousand Oaks, CA: Sage.

Harter, J. K., Schmidt, F. L., \& Hayes, T. L. (2002). Business-unit-level relationship between employee satisfaction, employee engagement, and business outcomes: A meta-analysis. Journal of Applied Psychology, 87(2), 268-279.

Hauck, N., Filho, Machado, W. L., Teixeira, M. A. P., \& Bandeira, D. R. (2012). Marcadores reduzidos para a avaliação da personalidade em adolescentes. Psico-USF, 17(2), 253-261. doi: $10.1590 /$ S1413-82712012000200009

Heidemer, H., \& Moser, K. (2009). Self-other agreement in job performance ratings: A meta-analytic test of a process model. Journal of Applied Psychology, 94(2), 353-370. doi: 10.1037/00219010.94.2.353

Heller, K. A., \& Perleth, C. (2008). The Munich High Ability Test Battery (MHBT): A multidimensional, multimethod approach. Psychology Science Quarterly, 50(2), 173-188.

Helmreich, R. L., \& Spence, J. T. (1978). The Work and Family Orientation Questionnaire: An objective instrument to assess components of achievement motivation and attitudes toward family and career. Catalog of Selected Documents in Psychology, 8(2).

Hewlett, S. A. \& Rashid, R. (2011). Winning the War for Talent in Emerging Markets: Why Women Are the Solution. Boston, MA: Harvard Business Press.

Hodges, T. D., \& Clifton, D. O. (2004). Strengthsbased development in practice. In P. A. Linley $\&$ S. Joseph, International handbook of positive psychology in practice: From research to application (pp. 256-268). New York: Wiley.

Horn, J. L. (1965). A rationale and technique for estimating the number of factors in factor analysis. Psychometrika, 30(1), 179-185.

Hyde, J. S. (2005). The gender similarities hypothesis. American Psychologist, 60(6), 581-592. doi: 10.1037/0003-066x.60.6.581
Jackson, D. L., Gillaspy, J. A., Jr., \& Purc-Stephenson, R. (2009). Reporting practices in confirmatory factor analysis: An overview and some recommendations. Psychological Methods, 14(1), 6-23. doi: 10.1037/a0014694

Kaiser, H. F. (1960). The application of electroniccomputers to factor-analysis. Educational and Psychological Measurement, 20(1), 141-151. doi: $10.1177 / 001316446002000116$

Marsh, H. W., Hau, K., \& Wen, Z. (2004). In Search of Golden Rules: Comment on Hypothesis-Testing Approaches to Setting Cutoff Values for Fit Indexes and Dangers in Overgeneralizing $\mathrm{Hu}$ and Bentler's (1999). Findings. Structural Equation Modeling: A Multidisciplinary Journal, 11(3), 320-341. doi: 10.1207/s15328007sem1103_2

Martin, J., \& Smith, C. (2010). How to keep you top talent. Harvard Business Review, 88(5), 54-61.

McDonnell, A. (2011). Still Fighting the "War for Talent"? Bridging the Science versus Practice Gap. Journal of Business and Psychology, 26(2), 169-173. doi: 10.1007/s10869-011-9220-y

Michaels, E., Handfield-Jones, H., \& Axelrod, B. (2001). The war for talent. Boston, MA: Harvard Business School Press.

Nakano, T. C., \& Siqueira, L. G. G. (2012). Validade de conteúdo da Gifted Rating Scale (versão escolar) para a população brasileira. Avaliação Psicológica, 11(1), 123-140.

Nunes, C. H. S. S., Hutz, C. S., \& Nunes, M. F. O. (2010). Bateria Fatorial de Personalidade. São Paulo, SP: Casa do Psicólogo.

Nunnally, J. C. (1967). Psychometric theory. New York: McGraw Hill.

O'Boyle, E., \& Aguinis, H. (2012). The best and the rest: Revisiting the norm of normality of individual performance. Personnel Psychology, 65, 79-119. doi: 10.1111/j.1744-6570.2011.01239.x

Pasquali, L. (2005). Análise fatorial para pesquisadores. Brasília, DF: Laboratório de Pesquisa em Avaliação e Medida, Universidade de Brasília.

Pepermans, R., Vloeberghs, D., \& Perkisas, B. (2003). High potential identification policies: An empirical study among Belgian companies. Journal of Management Development, 22(8), 660-678. doi: 10.1108/02621710310487846

Peterson, C., \& Seligman, M. E. P. (2004). Character strengths and virtues: A handbook and classification. New York: Oxford University Press. 
Ready, D. A., Conger, J. A., \& Hill, L. A. (2010). Are you a high potential? Harvard Business Review, $88(6), 78-84$.

Seligman, M. E. P., \& Csikszentmihalyi, M. (2000). Positive psychology: An introduction. American Psychologist, 55, 5-14. doi: 10.1037/0003066X.55.1.5

Silzer, R., \& Church, A. H. (2009). The potential of potential. Industrial and Organizational Psychology, 2, 446-452.
Simon, H. A., \& Chase, W. G. (1973). Skill in chess. American Scientist, 61, 394-403.

Ulrich, D. (2006). The talent trifecta. Workforce Management, 10, 32-33.

\section{Anexo}

\section{Escala de Talento nas Organizações}

Abaixo há uma lista de afirmativas que dizem respeito a situações que podem ocorrer com as pessoas no ambiente de trabalho. Solicitamos que você leia cada uma das frases e responda na escala ao lado delas o quanto você concorda que elas dizem respeito a você. Leve em consideração que o número 1 da escala significa que você discorda totalmente da frase e o número 5 que você concorda totalmente.

\begin{tabular}{|c|c|c|c|c|c|}
\hline & $\begin{array}{c}\text { Discordo } \\
\text { totalmente } \\
1\end{array}$ & 2 & $\begin{array}{c}\text { Nem concordo, } \\
\text { nem discordo } \\
3\end{array}$ & 4 & $\begin{array}{c}\text { Concordo } \\
\text { totalmente } \\
5\end{array}$ \\
\hline $\begin{array}{l}\text { 1. Geralmente me destaco em minha equipe pela } \\
\text { boa qualidade do meu trabalho. }\end{array}$ & 1 & 2 & 3 & 4 & 5 \\
\hline $\begin{array}{l}\text { 2. Costumo ouvir que demoro para fazer minhas } \\
\text { atividades de trabalho. }\end{array}$ & 1 & 2 & 3 & 4 & 5 \\
\hline $\begin{array}{l}\text { 3. Frequentemente sou capaz de encontrar sozinho } \\
\text { soluções para problemas de trabalho. }\end{array}$ & 1 & 2 & 3 & 4 & 5 \\
\hline $\begin{array}{l}\text { 4. Costumo ouvir que sou melhor do que outras } \\
\text { pessoas que fazem atividades semelhantes à minha. }\end{array}$ & 1 & 2 & 3 & 4 & 5 \\
\hline $\begin{array}{l}\text { 5. Geralmente consigo fazer meu trabalho sem } \\
\text { precisar de ajuda. }\end{array}$ & 1 & 2 & 3 & 4 & 5 \\
\hline $\begin{array}{l}\text { 6. Costumo demorar mais do que outras pessoas } \\
\text { para fazer o meu trabalho. }\end{array}$ & 1 & 2 & 3 & 4 & 5 \\
\hline $\begin{array}{l}\text { 7. Com frequência as pessoas ficam admiradas } \\
\text { com a qualidade do meu trabalho. }\end{array}$ & 1 & 2 & 3 & 4 & 5 \\
\hline $\begin{array}{l}\text { 8. Costumo atrasar para concluir minhas atividades } \\
\text { de trabalho. }\end{array}$ & 1 & 2 & 3 & 4 & 5 \\
\hline $\begin{array}{l}\text { 9. Soluciono problemas de trabalho sem ajuda de } \\
\text { outras pessoas. }\end{array}$ & 1 & 2 & 3 & 4 & 5 \\
\hline
\end{tabular}




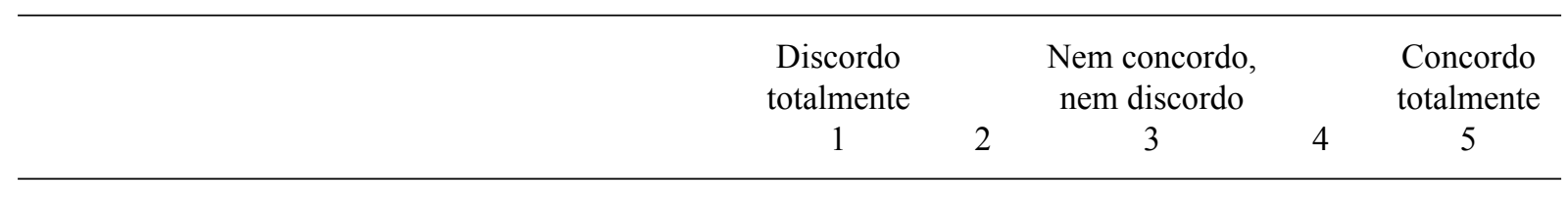

10. Posso ser considerado o número 1 no trabalho que faço.

1

2

3

4

5

11. Costumo concluir minhas atividades de trabalho no último minuto possível.

1

2

3

4

5

12. Sinto que tenho algum talento especial que me faz realizar bem meu trabalho.

1

1

2

3

45

13. Meus colegas de trabalho dizem que eu sou bom no que faço.

1

2

3

45

14. Levo mais tempo que as outras pessoas
normalmente levam para fazer meu trabalho.
15. Geralmente consigo fazer meu trabalho sozinho.

16. Acho que o que falta para que eu seja o número 1 no que eu faço é concluir minhas atividades mais rapidamente.

17. Quando não sei como fazer alguma atividade de trabalho, consigo descobrir sozinho.

18. Meus superiores consideram meu desempenho melhor do que a média.

19. Consigo perceber sozinho como fazer um trabalho melhor.

20. Quando as pessoas precisam que alguma coisa seja feita com perfeição no trabalho buscam a minha ajuda.

Nota. Cálculo das Médias: Fator Qualidade: calcular média aritmética dos itens 1, 4, 7, 10, 12, 13, 18, 20. Fator Velocidade: inverter e calcular média aritmética dos itens: 2, 6, 8,11,14, 16. Fator Autonomia: calcular média aritmética dos itens 3, 5, 9, $15,17,19$. 